\title{
Malignant Tumors of Heart: A Review
}

\author{
Akshay Chauhan ${ }^{*}$, Anurag Agarwal' ${ }^{2}$, S. E. H. Naqvi², M. A. Geelani' \\ ${ }^{1}$ Department of CTVS, GB Pant Institute of Postgraduate Medical Education and Research, Jawaharlal Nehru Marg, \\ New Delhi, India \\ ${ }^{2}$ Department of CTVS, GBPH, New Delhi, India \\ Email: *dr.akshaychauhan1990@gmail.com
}

How to cite this paper: Chauhan, A., Agarwal, A., Naqvi, S.E.H. and Geelani, M.A. (2018) Malignant Tumors of Heart: A Review. World Journal of Cardiovascular Surgery, 8, 204-212.

https://doi.org/10.4236/wjcs.2018.811020

Received: October 19, 2018

Accepted: November 16, 2018

Published: November 19, 2018

Copyright $\odot 2018$ by authors and Scientific Research Publishing Inc. This work is licensed under the Creative Commons Attribution International License (CC BY 4.0).

http://creativecommons.org/licenses/by/4.0/

\begin{abstract}
Malignant cardiac tumors are rare and challenging entity. Experience in treating these aggressive and lethal tumors is minimal and management protocols are not well defined and have to rely on case reports, and personal experiences. Most effective therapy for primary cardiac malignancy is a complete surgical resection and the role of chemoradiotherapy is still evolving.
\end{abstract}

\section{Keywords}

Malignant Cardiac Tumors, Cardiac Sarcomas

\section{Open Access}

\section{Introduction}

Malignant cardiac tumors are a very rare entity and pose a unique diagnostic and therapeutic challenge. Most cardiologists and cardiac surgeons have minimal experience in treating these aggressive and lethal tumors. The first successful resection of a cardiac tumor was carried out by Crafoord in 1954 [1]. But still due to the absence of data from clinical trials, management protocols are not well defined and have to rely on case reports, and personal experiences. This article reviews histological malignant cardiac tumor subtypes and their management.

\section{Epidemiology}

The reported prevalence of cardiac tumors ranges from $0.17 \%$ to $0.28 \%$ in various autopsy series [2] [3] [4]. Slightly more frequently tumors of the heart occur in children. Simcha and Nadas reported the incidence of cardiac tumors in children as $0.08 \%$ and $0.027 \%$ respectively. Bussani reported decreasing occurrence with age $(16.8 \%$ in people $<64$ vs $8.5 \%$ in people $>85)$, which was attributed to less biological aggressiveness in the elderly [3] [4]. But the prevalence of cardiac neoplasms, unlike other malignancies, have shown little change over 
time. Among the primary cardiac tumors, about $10 \%-25 \%$ are malignant. Of the malignant tumors, about $75 \%-90 \%$ are sarcoma [5]. Angiosarcomas and unclassified sarcomas being the most common. Metastatic involvement of the heart is even more common with prevalence of $2.3 \%-18.3 \%$. Melanoma (hematogenous route), breast and lung carcinoma (direct infiltration and lymphatic route) show the incidence of cardiac metastasis [6].

\section{Histological Subtypes}

\subsection{Angiosarcoma}

Angiosarcomas account for almost $40 \%$ and the most common subgroup of primary malignant cardiac tumours. Incidence peaks in the middle age and no sex predilection. The most frequent location is the right atrioventricular groove and right atrial wall. Arterial phase enhancement on computed tomography (CT) and MRI is characteristic. It usually metastases to lungs and are the most aggressive cardiac tumors. Survival after diagnosis rarely exceeds 6 to 12 months. Grossly it is brownish and lobulated intramural neoplasm, and may infiltrate the pericardium. On histolopathology, they show an irregular, anastomosing, vascular network, lined by pleomorphic, atypical cells strongly positive with factor VIII, CD31 and CD34 [7].

\subsection{Malignant Pleomorphic Fibrous Histiocytoma (MFH)/Undifferentiated Pleomorphic Sarcoma}

This subtype accounts for almost one-third of all cardiac sarcomas. It is a diagnosis of exclusion, when all the immunohistochemical stains fail to give evidence of specific differentiation. Most frequently it arises in the atrium and left ventricle. They mimic left atrial myxoma with an endocavitary growth on echocardiography. Macroscopically it may be multiple, whitish with a rough surface and hard consistency, which differentiates it from myxomas. On MRI, infiltrative growth into the atrial wall and lack of attachment site on the atrial septum further differentiates them [7].

\subsection{Leiomyosarcoma}

Leiomyosarcoma is sarcoma of smooth muscle cells of endocardium with a peak in the $5^{\text {th }}$ decade ( $10 \%$ of all cardiac malignancies). Usually occurs in the left atrial roof as multiple endocavitary mass and the pulmonary infundibulum and artery, where it presents like pulmonary embolism. Tumor smooth muscle cells are actin and desmin positive [8].

\subsection{Fibrosarcoma}

This subtype consists of a malignant proliferation of mesenchymal cells with fibroblastic features. They usually occur in adults with atria as the most common site. Pericardial form of this subtype presents like a mesothelioma. Microscopically, the fibrosarcoma consists of a collagen stroma and monomorphic spindle 
cells, positive for vimentin and actin. They represent nearly $5 \%$ of all primary cardiac malignancies and the prognosis is poor (mean survival 5 months) [9].

\subsection{Rhabdomyosarcoma}

Rhabdomyosarcoma is an aggressive malignant tumour of striated muscle ( $0 \%$ to $5 \%$ of primary cardiac sarcomas), most frequently encountered in children at a mean age of approximately 14 years. It has a predilection for the ventricle and diffusely infiltrates the myocardium. PAS positive rhabdomyoblasts (positive for vimentin, muscle-specific actin, desmin and myogenin) with typical "tadpole" appearance are characteristic on histopathology. Prognosis is poor [10].

\subsection{Liposarcoma}

Liposarcoma are extremely rare (1\% of primary malignant tumours of the heart) and mimic a atrial myxoma, with early signs of local invasion. Grossly, it appears as intramural, sessile or pedunculated mass, gelatinous and un-encapsulated. Microscopically, it consists of lipoblasts, which are S100 positive [11].

\subsection{Cardiac Lymphoma}

It represents $5 \%$ of primary cardiac malignant neoplasms. It usually occurs in the elderly with a male preponderance. It usually occurs as a infiltrating intramural mass in the right atrium, extending to pericardium with massive effusion. It is an extranodal non-Hodgkin's lymphoma. $80 \%$ are B-cell subtype with CD20 positive cells, and rest are CD3 positive T-cell lymphomas. They should be promptly treated with chemotherapy, and with early treatment have a good prognosis [12].

\section{Clinical Presentation}

\subsection{General Symptoms}

Lymphomas usually present by a low grade fever, weight loss, myalgia and night sweats due to secretion of interleukins and various tumor cell factors. Hemorrhagic pericardial effusion is frequently associated with malignant cardiac tumors.

\subsection{Obstructive Symptoms}

Tumors in atria may restrict the movement of atrioventricular valves, producing symptoms of stenosis or regurgitation of the mitral or tricuspid valve. Mobile, pediculated neoplasms thus generally lead to dyspnea, depending on posture. Infiltration of tumor mass into ventricular wall may produce restrictive cardiomyopathy. Expansion of tumor may even obstruct the venae cavae and produce superior vena cava syndrome. Small intramural tumors rarely produce symptoms. Metastatic disease from other organs usually affect the pericardium causing pericardial effusion, cardiac tamponade, or constrictive pericarditis [7]. 


\subsection{Arrhythmias}

Infiltration of the neural pathways can cause irregular heartbeat and various degrees of heart block especially in fibromas. This may even result in sudden cardiac death.

\subsection{Embolism}

Stroke, embolism of the peripheral vasculature, or a pulmonary artery embolism, can be caused by detached friable tumor tissue or thrombotic deposits over tumor surface. Predisposition to embolic episodes depends mainly on the type of the tumor, its location (intramural or intracardial), and the fragility.

\subsection{Metastasis}

Most frequently, sarcomas metastases affect lungs, brain, and bones [10].

\section{Investigations}

\subsection{ECG}

It is usually nonspecific in most cases. Rarely may demonstrate shortening of $\mathrm{P}$ $\mathrm{R}$ interval, the right or left bundle branch block (RBBB or LBBB) or complete heart block.

\subsection{Chest X-Ray}

Heart shadow maybe enlarged (cardiomyopathy, pericardial effusion) or asymmetrical enlargement of the heart may occur when the tumor is located within the anterolateral wall of left ventricle. Calcifications maybe present in rhabdomyoma and liposarcoma.

\subsection{Echocardiography}

Echocardiography has a high sensitivity and specificity (90\% and 95\%, resp.) for the detection of cardiac tumors. Transthoracic echocardiography (TTE) can visualize the shape, size, extent and relation of the tumor to adjacent cardiac structures. Hemodynamic consequences of the tumor can also be studied. Transesophageal echocardiography (TEE) is superior to TTE in identifying small tumors $(<5 \mathrm{~mm})$ and tumors localized at the posterior cardiac segments [13]. Contrast echocardiography helps in the differentiating tumors by examining tissue perfusion. Malignant tumors or tumors rich in vascularity, appear with an intense enhancement of the echocardiographic image. Benign cardiac tumors exhibit sparse vascularity. Three dimensional echocardiography (3D Echo) contributes mainly to an improved assessment of the shape, the size, the mobility of a tumor and its relationship regarding adjacent structures, by making use of the wider imaging range that this technique provides [14].

\subsection{Magnetic Resonance}

It is required for a better assessment of the tumor relation to adjacent structures, 
in order for a surgical resection technique to be planned. Myocardial infiltration, expansion of the mass to the pericardium or to adjacent structures and tumor composition can be studied. Administration of contrast medium helps differentiate a cardiac tumor from thrombus [15].

\subsection{Computed Tomography}

It can also provide useful information, due to its high resolution and its ability to accurately depict cardiac morphology. Disadvantages include less information regarding characterization of tissues in comparison to MRI apart from associated radioactivity and of nephrotoxicity of contrast mediums. Multidetector computed tomography (MDCT) is useful for detecting small lesions and staging of malignant tumors due to its high spatial resolution. Calcification and fat content within a mass can also be assessed [16].

\subsection{Positron Emission Tomography (PET)}

Useful in identifying cardiac involvement in patients with metastatic tumors and has a sensitivity of over $90 \%$ in differentiating benign and malignant tumors [17].

\subsection{Coronary Angiography}

Used assess tumor vascularity and the coronary infiltration especially in angiosarcomas. Helps in decision making and planning for surgery.

\subsection{Histological Evaluation}

Histological confirmation is necessary for making diagnosis of cardiac malignancy and estimation of grade. This can be achieved with cytological examination of pericardial fluid or image guided percutaneous or transvenous cardiac biopsy. If diagnosis cannot be established, thoracoscopic biopsy may be needed. Biopsy is considered reasonable if potential benefits outweigh risks.

\section{Treatment}

Cardiac malignancy patients should be treated at an interdisciplinary center where oncologists, radiotherapists, and surgeons cooperate closely. Cardiac tumors present a particular challenge for heart surgeons. There is no evidence based optimal treatment regime or guidelines for treatment of cardiac malignancies, as it's a rare disease.

\subsection{Simple Tumor Resection}

Simple resection is usually possible only in benign tumors. Malignant tumors rarely present early so simple tumor resection is not possible. The tumor and its root is removed along with part of the septum and the defect is closed with patch material. Dislodging any tumor material is avoided [18]. All the chambers of the heart are inspected to exclude the presence of other tumors. This intervention is 
relatively uncomplicated.

\subsection{Complex Tumor Resection}

The greatest determinant of survival and most effective therapy for primary cardiac sarcomas is a complete surgical resection. This is possible only when mass has not infiltrated the adjacent tissues. So, complete removal is mainly determined by the anatomic location of and the structures involved by the tumor.

Tumors of the left heart are difficult to completely resect as often these are large tumors and anatomical exposure is inadequate, resulting in incomplete resection and rapid local recurrence. Left atrial tumors are typically approached through the interatrial groove. This may be satisfactory for small sized tumors but has inadequate exposure for large malignant tumors. Complete cardiectomy and orthotopic transplantation can be done in such cases [19].

Approach through the aortic and mitral valve is inadequate for complete resection of malignant tumors. Left ventriculotomy through viable muscle is not favored as it results in postoperative ventricular dysfunction.

Right heart sarcomas present with non-specific symptoms and less often cardiac failure, delaying the diagnosis. So, they are bulkier and present in more advanced stages with distant metastatic disease. In advanced tumors involving the right side of the heart hypothetically the whole right half of the organ can be resected. Pulmonary blood flow is maintained by Fontan circulation but chronic right heart failure may result in long term.

Tumors of pulmonary trunk often involve the pulmonary valve and extend distally along the artery but artery wall penetration is rare. Pulmonary root is excised and replaced with allograft and Dacron graft is used to extend to the first PA bifurcation [20].

\subsection{Autotransplantation}

In circumstances where tumor involves the posterior wall of the left atrium or the dorsal great vessels, the heart can be removed from the mediastinum to facilitate a complete surgical resection. Inadequate view of the tumor at conventional surgery, leads to incomplete resection and an early recurrence. This technique allows optimal visualization of all structures. Post-tumor resection the normal cardiac anatomy is restored using artificial materials (prostheses, patches, valves) or biological tissue and the heart is reimplanted. But this may result left and/or right heart failure due to excessive tumor resection [21]. Cardiac autotransplantation was first attempted by Cooley in 1984 for a left atrial paraganglioma.

\subsection{Artificial Heart}

If the tumor also involves a large area of left heart, and provided there are no metastases, the implantation of a total artificial heart can be considered as last resort in young patients. Some teams are exploring this modality as a bridge to chemotherapy and possible transplantation. External support ventricular assist 
devices and SynCardia Total Artificial Heart have been used in isolated cases [22].

\subsection{Cardiac Transplantation}

If distant metastases can be ruled out, transplantation can occasionally be considered as an option in non-resectable tumors. But, exacerbation of undetected micrometastases by the necessary immunosuppression remains a theoretical risk. Orthotopic transplantation for sarcoma have shown a median survival of only 12 months [23].

\subsection{Chemotherapy and Radiotherapy}

Adjuvant and neoadjuvant chemotherapy has been used at various centres to improve the poor results with resection alone. Rhabdomyosarcomas may have a better outcome with chemotherapy. Radiation has been used rarely, and mainly as a treatment of metastases. Non-sarcoma malignant tumors such as lymphoma and plasmacytomas respond very well to chemotherapy. Neoadjuvant chemotherapy for all primary sarcomas is used to reduce the tumor bulk and improve R0 resection rates and survival. Patients without heart failure are started on doxorubicin and ifosfamide. Chemotherapy is continued as long as a positive response is obtained. If the patient has obtained a significant response and metastatic disease is controlled, patient is taken up for surgical resection of the residual tumor. If the patient fails to respond to neoadjuvant chemotherapy or metastatic disease is not controlled, then only definitive chemotherapy and radiotherapy are considered [24].

\section{Prognosis}

The median survival time of 6 to 12 months of untreated cardiac sarcomas is extended to 17 to 24 months when complete surgical resection is done. Resection with positive margins has lower survival, with median survival time of 6 to 10 months. This emphasizes the importance of complete resection in these patients [25] [26].

\section{Conclusions}

Cardiac malignancies are rare and deadly. A multidisciplinary approach to all primary cardiac sarcomas is required with careful evaluation for local tumor extent as well as metastatic involvement. Radical resection, with or without postoperative chemotherapy, should be attempted whenever feasible. It may be considered as the gold standard of care.

It improves the long-term survival and quality of life for the patients but still new and innovative treatment strategies are required.

\section{Conflicts of Interest}

The authors declare no conflicts of interest regarding the publication of this pa- 
per.

\section{References}

[1] Chitwood, W.R. (1992) Clarence Crafoord and the First Successful Resection of a Cardiac Myxoma. The Annals of Thoracic Surgery, 54, 997-998. https://doi.org/10.1016/0003-4975(92)90676-U

[2] Kumar, S., Chaudhry, M.A., Khan, I., et al. (2004) Metastatic Left Atrial Synovial Sarcoma Mimicking a Myxoma. The Journal of Thoracic and Cardiovascular Surgery, 128, 756-758. https://doi.org/10.1016/j.jtcvs.2004.03.037

[3] Simcha, W., Wells, B.G. and Tynan, M.J. (1971) Primary Cardiac Tumors in Childhood. Archives of Disease in Childhood, 46, 508-514. https://doi.org/10.1136/adc.46.248.508

[4] Nadas, A.S. and Ellison, R.C. (1968) Cardiac Tumors in Infancy. Journal of Cardiology, 21, 363-366. https://doi.org/10.1016/0002-9149(68)90140-9

[5] Reardon, M.J., De Felice, C.A., Sheinbaum, R. and Baldwin, J.C. (1999) Cardiac Autotransplant for Surgical Treatment of a Malignant Neoplasm. The Annals of Thoracic Surgery, 67, 1793-1795. https://doi.org/10.1016/S0003-4975(99)00343-4

[6] Gunther, T., Schreiber, C., Noebauer, C., Eicken, A. and Lange, R. (2008) Treatment Strategies for Pediatric Patients with Primary Cardiac and Pericardial Tumors: A 30-Year Review. Pediatric Cardiology, 29, 1071-1076. https://doi.org/10.1007/s00246-008-9256-6

[7] Burke, A. (2008) Primary Malignant Cardiac Tumors. Seminars in Diagnostic Pathology, 25, 39-46. https://doi.org/10.1053/j.semdp.2007.10.006

[8] Donsbeck, A.V., Ranchere, D., Coindre, J.M., et al. (1999) Primary Cardiac Sarcomas: An Immunohistochemical and Grading Study with Longterm Follow-Up of 24 Cases. Histopathology, 34, 295-304. https://doi.org/10.1046/j.1365-2559.1999.00636.x

[9] Molina, J.E., Edwards, J.E. and Ward, H.B. (1990) Primary Cardiac Tumors: Experience at the University of Minnesota. The Journal of Thoracic and Cardiovascular Surgery, 38, 183-191. https://doi.org/10.1055/s-2007-1014064

[10] Burke, A., Jeudy Jr., J. and Virmani, R. (2008) Cardiac Tumours: An Update: Cardiac Tumours. Heart, 94, 117-123. https://doi.org/10.1136/hrt.2005.078576

[11] Bois, M.C., Bois, J.P., Anavekar, N.S., et al. (2014) Benign Lipomatous Masses of the Heart: A Comprehensive Series of 47 Cases with Cytogenetic Evaluation. Human Pathology, 45, 1859-1865. https://doi.org/10.1016/j.humpath.2014.05.003

[12] Jeudy, J., Kirsch, J., Tavora, F., et al. (2012) From the Radiologic Pathology Archives: Cardiac Lymphoma: Radiologic-Pathologic Correlation. Radiographics, 32, 1369-1380. https://doi.org/10.1148/rg.325115126

[13] Gulati, G., Sharma, S., Kothari, S.S., Juneja, R., Saxena, A. and Talwar, K.K. (2004) Comparison of Echo and MRI in the Imaging Evaluation of Intracardiac Masses. Cardiovascular and Interventional Radiology, 27, 459-469. https://doi.org/10.1007/s00270-004-0123-4

[14] Kaya, H., Gokdeniz, T., Tuncer, A. and Ozkan, M. (2010) Left Atrial Myxoma Demonstrated by Real-Time Three-Dimensional Transesophageal Echocardiography. Turk Kardiyoloji Dernegi Arsivi, 38, 222.

[15] O’Donnell, D.H., Abbara, S., Chaithiraphan, V., et al. (2009) Cardiac Tumors: Optimal Cardiac MR Sequences and Spectrum of Imaging Appearances. American Journal of Roentgenology, 193, 377-387. https://doi.org/10.2214/AJR.08.1895 
[16] Kim, E.Y., Choe, Y.H., Sung, K., Park, S.W., Kim, J.H. and Ko, Y.H. (2009) Multidetector CT and MR Imaging of Cardiac Tumors. Korean Journal of Radiology, 10, 164-175. https://doi.org/10.3348/kjr.2009.10.2.164

[17] Rahbar, K., Seifarth, H., Schafers, M., et al. (2012) Differentiation of Malignant and Benign Cardiac Tumors Using 18F-FDG PET/CT. Journal of Nuclear Medicine, 53, 856-863. https://doi.org/10.2967/jnumed.111.095364

[18] Hoffmeier, A., Schmid, C., Deiters, S., et al. (2005) Neoplastic Heart Disease-The Muenster Experience with 108 Patients. The Journal of Thoracic and Cardiovascular Surgery, 53, 1-8. https://doi.org/10.1055/s-2004-830389

[19] Hoffmeier, A., Deiters, S., Schmidt, C., et al. (2004) Radical Resection of Cardiac Sarcoma. The Journal of Thoracic and Cardiovascular Surgery, 52, 77-81. https://doi.org/10.1055/s-2004-817809

[20] Baker, P.B. and Goodwin, R.A. (1985) Pulmonary Artery Sarcomas. A Review and Report of a Case. Archives of Pathology \& Laboratory Medicine, 109, 35-39.

[21] Ramlawi, B., Al-Jabbari, O., Blau, L.N., et al. (2014) Autotransplantation for the Resection of Complex Left Heart Tumors. The Annals of Thoracic Surgery, 98, 863-868. https://doi.org/10.1016/j.athoracsur.2014.04.125

[22] Ried, M., Rupprecht, L., Hirt, S., et al. (2010) Sequential Therapy of Primary Cardiac Lymphoma with Cardiectomy, Total Artificial Heart Support, and Cardiac Transplantation. The Journal of Heart and Lung Transplantation, 29, 707-709. https://doi.org/10.1016/j.healun.2010.01.014

[23] Überfuhr, P., Meiser, B., Fuchs, A., et al. (2002) Heart Transplantation: An Approach to Treating Primary Cardiac Sarcoma? The Journal of Heart and Lung Transplantation, 21, 1135-1139. https://doi.org/10.1016/S1053-2498(02)00409-6

[24] Llombart-Cussac, A., Pivot, X., Contesso, G., Rhor-Alvarado, A., Delord, J.P., Spielmann, M., et al. (1998) Adjuvant Chemotherapy for Primary Cardiac Sarcomas: The IGR Experience. British Journal of Cancer, 78, 1624-1628.

https://doi.org/10.1038/bjc.1998.733

[25] Simpson, L., Kumar, S.K., Okuno, S.H., et al. (2008) Malignant Primary Cardiac Tumors: Review of a Single Institution Experience. Cancer, 112, 2440-2446. https://doi.org/10.1002/cncr.23459

[26] Putnam, J.B., Sweeney, M.S., Colon, R., Lanza, L.A., Frazier, O.H. and Cooley, D.A. (1991) Primary Cardiac Sarcomas. The Annals of Thoracic Surgery, 51, 906-910. https://doi.org/10.1016/0003-4975(91)91003-E 